\title{
Analysis of structural distortion in Eshelby twisted InP nanowires by scanning precession electron diffraction
}

\author{
Daniel Ugarte ${ }^{1,2}(\bowtie)$, Luiz H. G. Tizei ${ }^{3}$, Monica A. Cotta ${ }^{1}$, Caterina Ducati ${ }^{2}$, Paul A. Midgley ${ }^{2}$, and Alexander \\ S. Eggeman ${ }^{2,4}(\bowtie)$
}

${ }^{1}$ Instituto de Física "Gleb Wataghin", Universidade Estadual de Campinas-UNICAMP, CEP 13083-859, Campinas - SP, Brazil

${ }^{2}$ Department of Materials Science and Metallurgy, University of Cambridge, Cambridge CB3 OFS, UK

${ }^{3}$ Laboratoire de Physique des Solides, CNRS UMR8502, Univ. Paris Sud, 91405 Orsay, France

${ }^{4}$ School of Materials, University of Manchester, Manchester, M13 9PL, UK

(C) The Authors(s) 2019.

Received: 11 October 2018 / Revised: 17 January 2019 / Accepted: 4 February 2019

\begin{abstract}
Transmission electron microscopes (TEM) are widely used in nanotechnology research. However, it is still challenging to characterize nanoscale objects; their small size coupled with dynamical diffraction makes interpreting real- or reciprocal-space data difficult. Scanning precession electron diffraction ((S)PED) represents an invaluable contribution, reducing the dynamical contributions to the diffraction pattern at high spatial resolution. Here a detailed analysis of wurtzite $\mathrm{InP}$ nanowires (30-40 nm in diameter) containing a screw dislocation and an associated wire lattice torsion is presented. It has been possible to characterize the dislocation with great detail (Burgers and line vector, handedness). Through careful measurement of the strain field and comparison with dynamical electron diffraction simulations, this was found to be compatible with a Burgers vector modulus equal to one hexagonal lattice cell parameter despite the observed crystal rotation rate being larger (ca. 20\%) than that predicted by classical elastic theory for the nominal wire diameter. These findings corroborate the importance of the (S)PED technique for characterizing nanoscale materials.
\end{abstract}

\section{KEYWORDS}

electron microscopy, scanning precession electron diffraction, Eshelby twist, screw dislocation, nanowire, indium phosphide

\section{Introduction}

The ability to target and control specific chemical or physical properties have made nanostructured materials an essential constituent of many technological devices, such as sensors, electronic or optical components, photocells [1]. The huge surface-volume ratio gives rise to numerous chemical and physical properties as a function of size, chemical composition, atomic structure, etc. As the number of application opportunities have grown, the demand for more precise and sensitive characterization has driven technical development in instrumentation and methods. For example, semiconductor nanowires (NWs) are now proposed for many optoelectronic systems, and the understanding of their properties require precise measurement of chemical composition, doping, interface quality (structural and chemical), atomic structure distortions due to surface, interfaces and defects, etc. [1-3].

Transmission electron microscopes are widely used in the characterization of nanomaterials because the ability to produce electrons with a very short wavelength $(0.02 \AA$ at $300 \mathrm{kV}$ accelerating voltage) means that structural information at the atomic scale is readily accessible, the Lorentz force means that electrons can be focused to form sub-nanometre probes providing extremely high spatial resolution and the strong coulombic interaction between the beam and sample makes the microscope sensitive to light elements and tiny structural differences [4-7]. However, the strong interaction also gives rise to the phenomenon of dynamical diffraction, where the electron can be scattered multiple times as it traverses even a thin sample (unlike X-ray and neutron diffraction that assumes single or kinematical scattering [8]). This makes interpretation of the intensity of diffracted beams or the contrast in atomic resolution images difficult or impossible, since the scattering is not simply related to the underlying crystal potential $[5,7]$.

One solution to this difficulty is the use of precession electron diffraction (PED) [9], which integrates a volume of reciprocal space to form the diffraction pattern (compared to a single section in a conventional pattern). This helps to reduce the contribution of multi-beam dynamical scattering to the diffracted intensity, reduces the contribution of inelastic and incoherent background and also increases the number of reflections typically found in a pattern [10, 11]. These all help to make it a widely used application in structural characterization of materials. Of particular interest is the capability to combine PED with beam scanning, allowing the structure of a sample to be analyzed point by point across a large area at step sizes down to $1 \mathrm{~nm}$ [12]. This approach will be described as high spatial resolution PED or HSR-PED.

The spatial resolution and sensitivity to crystal distortions have made HSR-PED a powerful technique for studying strain and defects in materials [13-15] compared to image-based methods [16-19]. For this study a particularly complex system was analyzed, an indium phosphide [20] NW of radius $\sim 30 \mathrm{~nm}$ containing a screw dislocation along the wire axis. The crystal structure of the wires is wurtzite $\left(P 6_{3} m c, a=b=4.150 \AA, c=6.912 \AA\right)$, a phase that is not stable in macroscopic InP crystals. Energy minimization requires the dislocation core to be aligned with the wire axis [21]. The displacement field 
$u$ of a screw dislocation (see Fig. 1(a)) in an isotropic medium is non-zero only along $z$ axis $\left(u_{z} \neq 0, u_{x}=u_{y}=0\right)$, and the $u_{z}$ value depends only on the $x, y$ coordinate. In cylindrical coordinates, it results $u_{z}=B \theta /(2 \pi)$ (where $\theta$ is the azimuthal angle and, $B$ is the Burgers vector modulus $|\boldsymbol{B}|)$. This leads to a shear strain along the $\hat{\theta}$ versor $\gamma_{\theta z}=B /(2 \pi r)$ that depends linearly on the Burgers vector and inversely proportional to the distance to the dislocation line.

In these terms, the basal planes in the wurtzite crystal form a helicoid of pitch $B$ as shown in Fig. 1(a). This schema also shows graphically that the normal to the helicoid plane $(N)$ is tilted in relation to the wire geometrical axis $\left(\Delta N=N_{\theta} \hat{\theta}\right)$; the tilt shows opposite sign for radially opposite sites and will vary inversely proportionally to the radial distance from the dislocation core.

The preceding discussion has considered a cylinder fixed at the ends, such that it cannot twist. As demonstrated by Eshelby in 1950s, if the cylinder is unconstrained, the crystal will develop a backward torsion to cancel the torque created by the screw dislocation $[21,22]$. Using classical elastic theory and considering a wire of radius $R$, the torsion displacement field should be $u_{\theta}^{T}=-\alpha r z$, where the twist rate $\alpha$ results $\alpha=B /\left(\pi R^{2}\right)$ for a cylindrical wire. This phenomenon is described as the "Eshelby twist", and was experimentally observed for the first time in 2008 in PbSe and PbS NWs [23, 24]; and subsequently observed in NWs of different materials [25-29]. Within a PED orientation map, the twist should easily revealed as a continuous rotation of the crystal normal (ND) around the $c$-axis; this is schematically displayed at the right of Fig. 1(b) by the basal plane rotation (spinning wurtzite basal plane vectors, $\boldsymbol{a}, \boldsymbol{b}$, and $\boldsymbol{d}$ ).

In most of the previous studies [25-29], attempts to characterize the strain field in such NWs using conventional electron microscopy have concentrated on the twist rate measurements, yet these studies have proven at best inconsistent. The absolute twist can be quite easily determined using bright-field imaging to indicate the positions of

(a)
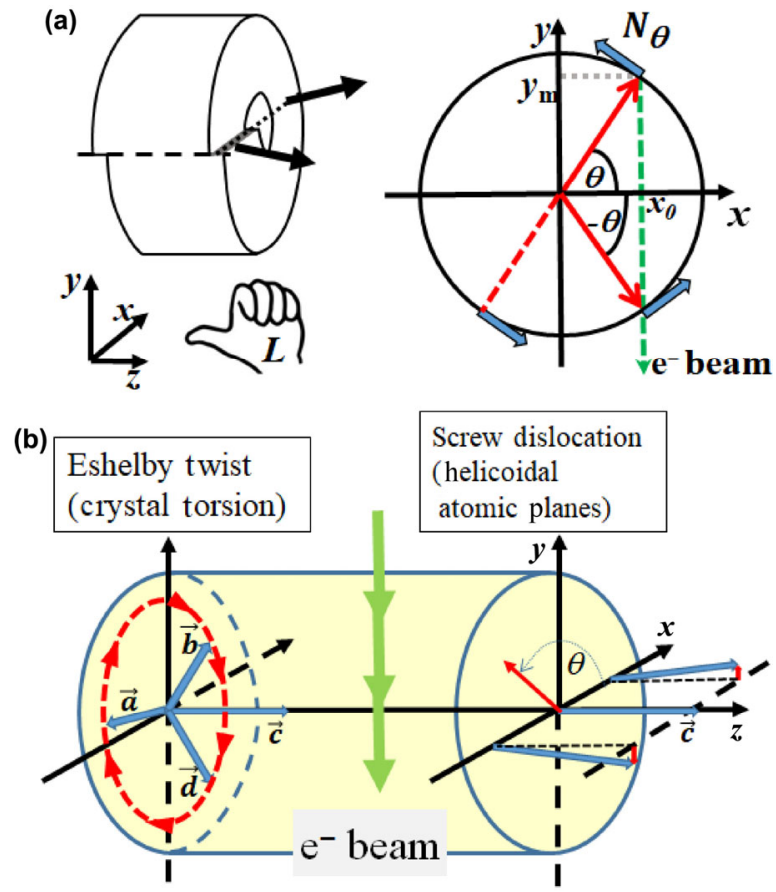

Figure 1 (a) Schematic drawing showing the occurrence of an axial left-handed screw dislocation in a nanowire (a hole has been placed at the wire center to avoid singularities); note the atomic planes form a helicoid; then the basal plane normal (i.e. the $c$-axis) shows a different orientation in opposite radial positions. (b) The distortion of crystal structure of a NW containing an axial screw dislocation includes two different contributions. Firstly, the helicoidal basal planes generate a disorientation of the $c$-axis which depends on the radius (right side of the cylindrical wire). Secondly, as predicted by Eshelby, a torsion must be generated to compensate the screw dislocation torque, represented by a continuous rotation of the basal plane base vectors ( $\boldsymbol{a}, \boldsymbol{b}$, and $\boldsymbol{d}$ outlined at left NW side). major zone-axis orientations along the wire, however in most cases the twist rate is appreciably larger than that predicted from the elasticity theory indicated previously. The advantage of HSR-PED is that the strain field can also be studied radially, or at least as a function of the lateral distance of the electron beam from the dislocation core $\left(x_{0}\right.$ in the schema in Fig. 1(a)) or impact parameter. For the InP wires studied in this article the wurtzite $c$-axis (i.e. helicoid normal) can be used to determine the misorientation to the wire axis and hence give a measure of the dislocation strain field independent of the twist rate. The limitation of angular resolution in the measurement is a potential limitation however. The Burgers vector of the screw dislocation should be one lattice parameter $(|\boldsymbol{c}|)$, hence the expected misorientation is $\sim 2^{\circ}$ at the central plane of the wire $(y=0)$ and approximately $5 \mathrm{~nm}$ from the central axis. This means that the measurement of the dislocation strain field will be very challenging, because even a small precession angle in the range of $0.3^{\circ}-0.5^{\circ}$ will lead to an averaging, or uncertainty, of about $1^{\circ}$ for the crystal orientation estimation.

To summarise, the presence of the screw dislocation creates a complex 3D distortion of the wire crystal structure. The Eshelby twisted NWs represent an interesting and stimulating case to test the ability of local strain analysis using PED-based maps of individual nanostructures.

\section{Results}

\subsection{Twist rate}

Using HSR-PED, it was possible to determine the crystal orientation at positions every $4 \mathrm{~nm}$ across a section of the wire. For the twist rate determination it is necessary to determine the vector component (hki0) parallel to the wurtzite basal plane. From a practical point of view it was found that the wire should not be aligned perpendicular to the electron beam direction. Although this gives the most direct access to the required vector component (since the $c$-axis should perpendicular to the beam direction), it was found that there were significant errors when determining the wire orientation by HSR-PED

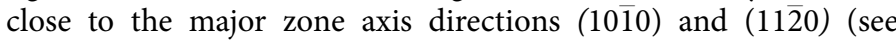
Fig. S5 in the Electronic Supplementary Material (ESM)). In order to get a more accurate orientation analysis the wire was tilted by a few degrees to avoid these major zone-axis directions and the vector component ( $h k i 0)$ was determined from the overall vector ( $h k i l$ ) parallel to the electron beam.

There was an additional benefit of this method for determination of the twist rate, since the crystallographic $c$-axis direction lies along the wire axis [30], and the component of the vector ( $h k i l$ ) parallel to (0002) provides information about the tilt of the wire axis with respect to the electron beam direction (shown in Fig. S3 in the ESM). This was initially used to account for the applied tilt of the NW but it also allowed any out of plane bending of the wire to be determined. Figure 2(a) shows a virtual image of the sample formed from the integrated intensity in the HSR-PED patterns, and shows how the wire bends in the $x-z$ plane. Furthermore after analysis of the orientation data the out of plane bending could also be accounted for, providing the full three-dimensional (3D) shape of the wire (see ESM for more details on this process). This allowed the true length of the wire in the images to be determined, as shown in Fig. 2(c) alongside the wire length measured from the $2 \mathrm{D}$ projection only. This discrepancy is one of the possible sources of error in the twistrate calculation so is a vital part of accurately characterizing the strain field and hence the nature of the dislocation in this NW.

To calculate the twist rate a section of the NW lying entirely across a hole in the carbon support film was chosen (to provide the clearest diffraction signals possible), and the corresponding virtual annular dark field (VADF) image is shown in Fig. 3(a). The average vector along a 7 pixel line parallel to the wire central line in the 
(a)

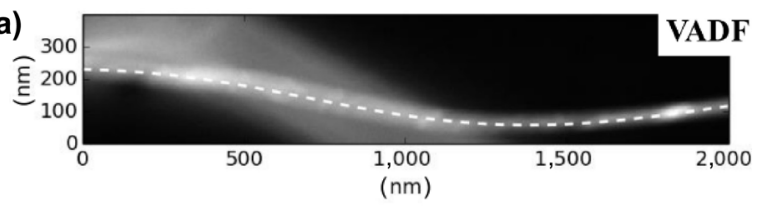

(b)
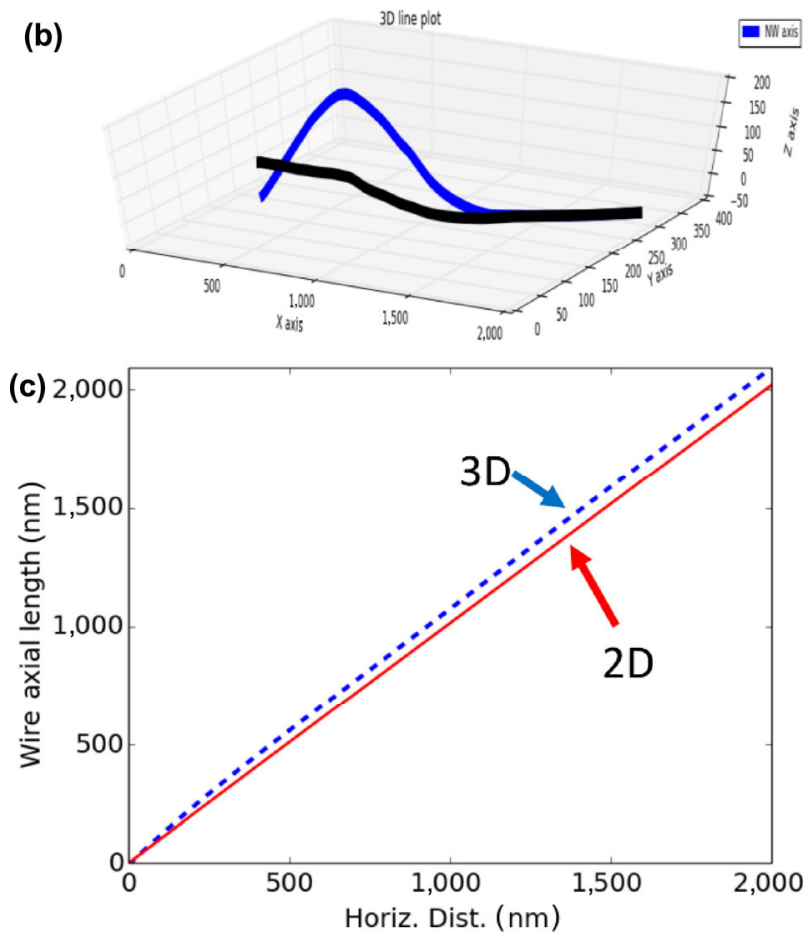

Figure 2 Analysis of the nanowire 3D bending on the electron microscopy sample as derived from HSR-PED data. (a) VADF image of the InP nanowire; the dashed line indicates the wire center. (b) Comparison of the deduced nanowire shape considering the VADF image (2D projection) and 3D shape (blue line deduced from the crystallographic orientation of central pixel along the wire length). (c) Comparison of the nanowire axial length obtained from the $2 \mathrm{D}$ and 3D deduced NW shapes; this distance must be used subsequently for the twist rate estimation.

image was determined and the disorientation (w.r.t to the left hand end of the wire section in Fig. 3(a)) of the (hki0) components as a function of position along the wire are shown in Fig. 3(b). These lie within a range of $\pm 15^{\circ}$ since the symmetry of the NW leads to equivalent orientations every $30^{\circ}$. Figure $3(\mathrm{c})$ shows the absolute misorientation (note the pole figure inset showing the disorientation) as a function of position along the wire (corrected for any $180^{\circ}$ ambiguities and to ensure the rotation sense is preserved), from this it is clear that there is a remarkably consistent twist rate of $(0.070 \pm$ $0.002) \% \mathrm{~nm}$ along nearly $2 \mu \mathrm{m}$ of the wire. This torsion rate is plotted as positive in the sample coordinates where the wire growth axis (i.e. wurtzite [0001] $]_{\mathrm{B}}$ ) points towards the positive $z$-axis direction or the NW tip (Fig. 1). The Eshelby torsion vector must point in the opposite direction to the Burgers vector and so we deduce that the screw dislocation $\boldsymbol{B}$ points towards the $[0001]_{\mathrm{A}}$ direction (the growth direction of the InP crystal is shown in Fig. S1 in the ESM).

A very instructive representation of the PED-based twist data is presented in the form of animations where each frame include the real space measurement position, the PED pattern and the inverse pole figure of the crystal orientation (Videos ESM1 and ESM2). These animations compare data for well-oriented and disoriented NWs.

From the orientation maps it is also possible to estimate the NW diameter, allowing the theoretical twist rate for the Burgers vector length $|\boldsymbol{B}|=|\boldsymbol{c}|$. Using annular dark field (ADF) scanning TEM images, the value obtained was $R_{\mathrm{ADF}}=(16 \pm 1) \mathrm{nm}$ (see Fig. S2 in the ESM). The actual NW crystal size can also be estimated from
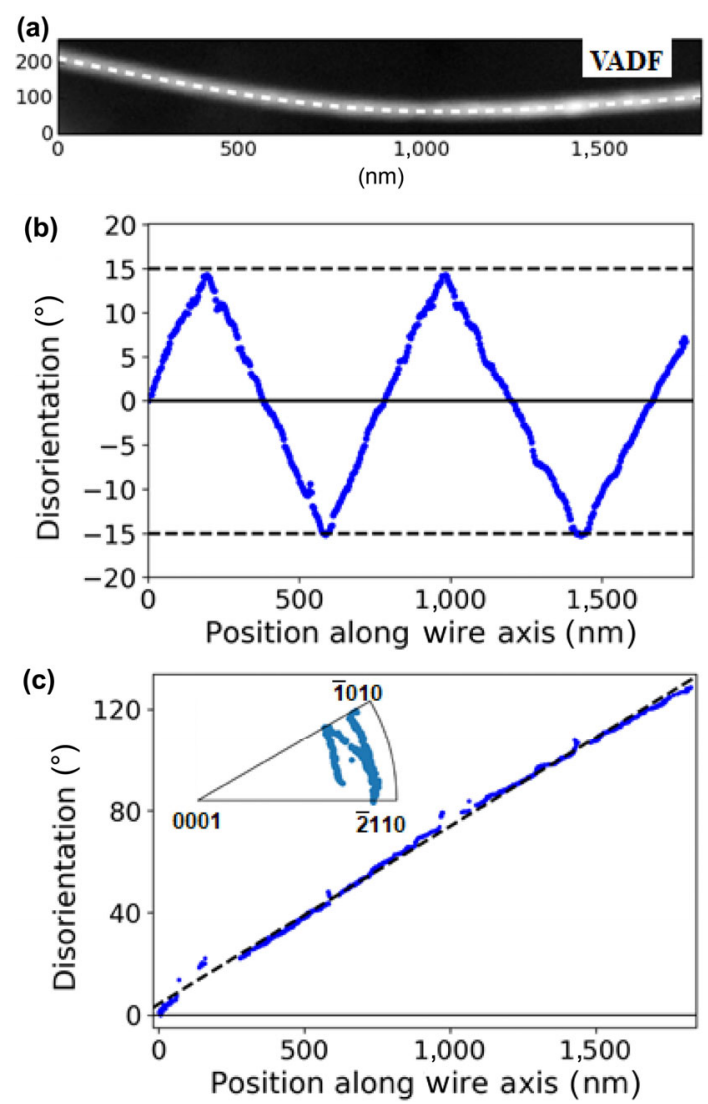

Figure 3 Analysis of Eshelby twist crystal rotation on the basis of HSR-PED data (a) Virtual dark field image of the nanowire; the line at the center of the wire indicates the wire axis position. (b) Change of orientation associated exclusively to the rotation of the basal plane component ( $h k i 0$ ) versus the position along wire axis. (c) Evolution of the NW crystal orientation along the wire axial coordinate (inset is the orientation projected on the reduced stereographic triangle).

PED data using individual diffraction patterns and PED fit quality factors. Considering this additional information, our best estimate of the wire radius becomes $R_{\mathrm{NW}}=(15 \pm 1) \mathrm{nm}$ (see Fig. S2 in the ESM). In this way, the expected twist rate should be $\alpha=(0.056 \pm$ $0.008) \% \mathrm{~nm}$, in contrast the experimentally PED derived twist value is ca. 1.2 times higher. From the point of view of the classical elastic theory, the wire supporting the experimentally measured torsion should be $\sim 13.5 \mathrm{~nm}$. It should be noted that the $\alpha$ error is rather high, mainly associated to the NW radius estimation uncertainty; which accounts for most of this discrepancy. The InP NWs may have a thin oxide and/or amorphous contamination layer at the surface and the presumably hexagonal NW cross section (rather than cylindrical) will lead to further reduction in the true diameter of the crystalline core of the NW compared to the image derived size. Such effects would increase the Eshelby twist rate further and bring the value within the error range of the experimental value. However study of these effects lie outside of the scope of the present study.

\subsection{Radial strain analysis}

In addition to the twist rate there is the possibility to verify the Burger's vector magnitude from the radial strain field analysis. To do this it is important to understand the meaning of the subtle changes of the $c$-axis orientation determined from PED data in relation to the wire geometrical axis. When a narrow electron beam traverses the wire (see schematic draw in Fig. 1(a)), it crosses crystal regions at different radial distances to the NW center. As the $c$-axis normal (indicated in Fig. 1(b)) depends on this distance and on the azimuthal position around the dislocation line, the HSR-PED measurement will be influenced by a range of different 
misorientations along the electron trajectory. To a first approximation, we might assume an averaging of misorientation along the electron trajectory in the resulting electron diffraction pattern (see Fig. S6 in the ESM). Figure 4 displays the distribution of angular misorientations for pixels over a region of ca. $64 \mathrm{~nm} \times 500 \mathrm{~nm}$ of the NW. This misorientation angle is in the $\hat{y}$ sense or, in our sample coordinates is positive (negative) for $x>0(x<0)$; which agrees with the $c$-axis vector positions in the schematic displayed in Fig. 1(b). This deviation distribution directly determines that the dislocation is left handed (see Fig. 1(a), where NW growth direction follows $\hat{z}$ ).

Figures 4(b) and 4(c) clearly show that the angular distribution clusters around $0^{\circ}$ if we analyze the deviations perpendicular to the electron beam, suggesting that any horizontal misorientation (parallel to $x$ ) above and below the $x-z$ plane is approximately compensated for; while each side of the wire show a different sign for the misorientation parallel to the electron beam. Even though the data clouds are rather broad $\left(\sim 1^{\circ}\right.$ close to the wire center, and $\sim 2^{\circ}$ at the wire surface) in Fig. 4(c), this data allows the form of the $c$-axis angular deviations due to the helicoidal structure of the basal planes to be determined. The misorientation varies smoothly from negative to positive through zero at the dislocation core, and it does not diverge at the dislocation core as predicted previously. One possibility was that the size of the electron beam caused averaging of misorientation information from both sides of the dislocation (Fig. S6(b) in the ESM).
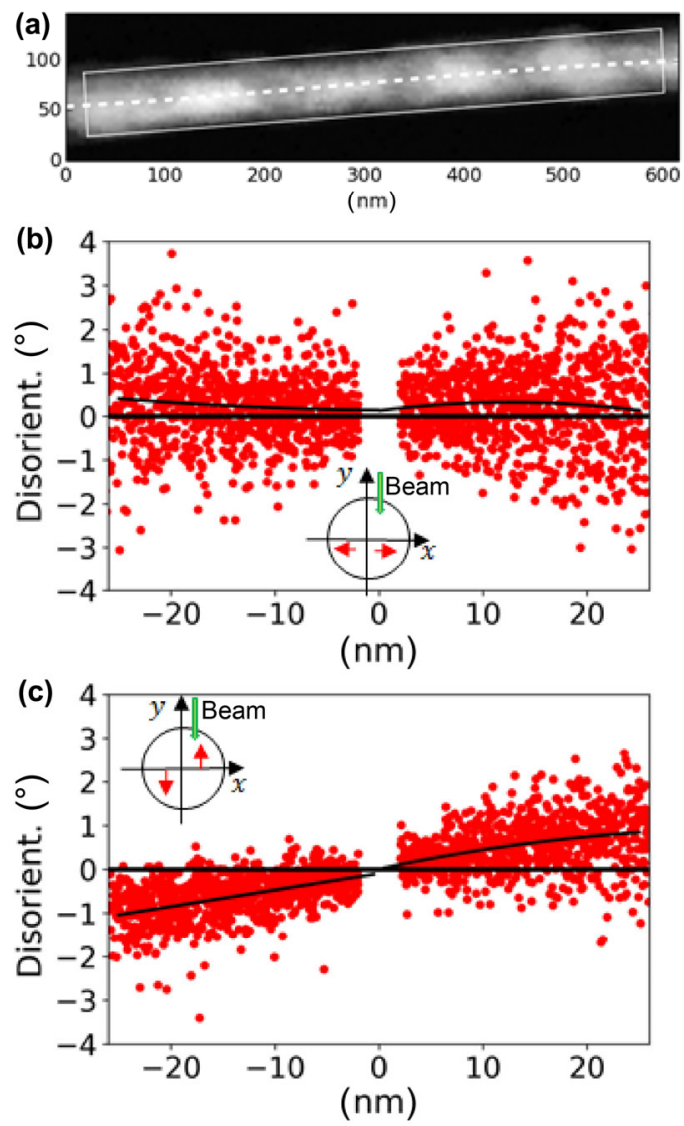

Figure 4 Analysis of the screw dislocation strain field. (a) VADF image of the InP nanowire (note the direction of sample coordinates, $z$ taken as the wire axis towards the right, $x$-axis points in upwards sense, and $y$-axis is perpendicular to the image plane). (b) Misorientation of the wurtzite $c$-axis in the plane perpendicular to the electron beam; we have plotted all pixels within the white rectangle in (a), with the exception of the central lines close to the dislocation core. The continuous black line indicates the average value as a function of the distance ( $x$ coordinate) to the dislocation core. (c) Misorientation in the plane parallel to the electron beam. Although the spread of the experimental points, it is clear that each side of the wire show disorientation of different signs as expected for a helicoidal atomic plane.
To remove this possibility additional experiments with improved spatial resolution (down to $1.25 \mathrm{~nm}$ ) were performed, with the results shown in Fig. 5. These show similar behavior to the results from the wider probe and the same conclusions can be drawn. Figure 5(c) shows that the $c$-axis misorientation still exhibits a transition from negative to positive at the dislocation core rather than the diverging in response to the average misorientation. It should be noted that the width of the transition region is considerably narrower indicating that spatial resolution is significantly improved. It is also significant that this NW region includes a portion of the crystal with an orientation very close to the $(11 \overline{2} 0)$ plane normal (marked with a dashed circle in Fig. 5(a)). Figure 5(d) shows the actual measured angle to the $x-z$ plane for each side across the dislocation line along beam direction (averaging the data clouds for each side of the dislocation) along the wire axis. It is obvious that the angular difference between wire sides is not constant and changes as the electron beam approaches different zone-axis directions along the wire. These results together suggest that the way that different crystal orientations influence the measured diffraction pattern is not uniform and so dynamical scattering needs to be considered if the measured misorientation is to be fully interpreted.

An atomic model of the NW containing a screw dislocation with $|\boldsymbol{B}|=|\boldsymbol{c}|$ was produced. Approximately $1 \mathrm{~nm} \times 1 \mathrm{~nm}$ sections through a model were used to generate scattering functions (phase gratings) for multislice simulations. The simulations were performed for impact parameters from 1-8 $\mathrm{nm}$ and for positions along the NW whose surface normal direction was between $9^{\circ}$ and $21^{\circ}$ from the (10 $\left.\overline{1} 0\right)$ pole towards $(11 \overline{2} 0)$.

The output from the dynamical simulations (example patterns are shown in Figs. 6(a) and 6(b)) was then subjected to the same orientation analysis as the experimental patterns. The misorientations to the simulated patterns for an impact parameter of 0 are shown in Fig. 6(c), which reproduce the general behaviour seen in the experimental results, with the misorientation of the $c$-axis normal decreasing as the impact parameter decreases. At higher angles the misorientation determined from the simulations tends towards a similar value to that seen from the experimental results, with an upper limit to the range of misorientations of approximately $2^{\circ}$ at an impact parameter of $8-10 \mathrm{~nm}$. In order to validate the magnitude of the Burger's vector, an additional set of simulation for $|\boldsymbol{B}|=2|\boldsymbol{c}|$ was performed for an impact parameter of $4 \mathrm{~nm}$. The calculated misorientation here was significantly larger than anything seen in the experimental results, supporting the conclusion that the actual magnitude was $|\boldsymbol{B}|=|\boldsymbol{c}|$.

\section{Discussion}

In accordance with our results, several studies have already reported that the Eshelby torsion in NWs maybe larger than that predicted by classical theory [23, 25-29]. The higher torsion rates have been associated by some authors with super-dislocations or linear defects with Burgers vectors larger than a unit cell lattice parameter [23]. In contrast, a previous study has concluded that the Eshelby relation is not followed in the case of InP wires, because the observed twist is not proportional to the Burgers vector modulus [25]. Another interpretation is that the associated dislocation has a Burgers vector whose modulus is an integer multiple of the lattice vector $n|c|$ ( $n$, integer $>1$, possibly $n=2$ ), although this seems unlikely given the ratio of experimental to theoretical twist rate is considerably less than two.

Accurately measuring the correct length of the wire in space is extremely helpful to correct for some of these effects but discrepancy in the estimated cross section area of the wire, possibly from surface disorder/oxidation/relaxation or error in the wires projected width mean that directly using the Eshelby formula to characterize the Burger's vector magnitude of the dislocation is difficult. 
(a)
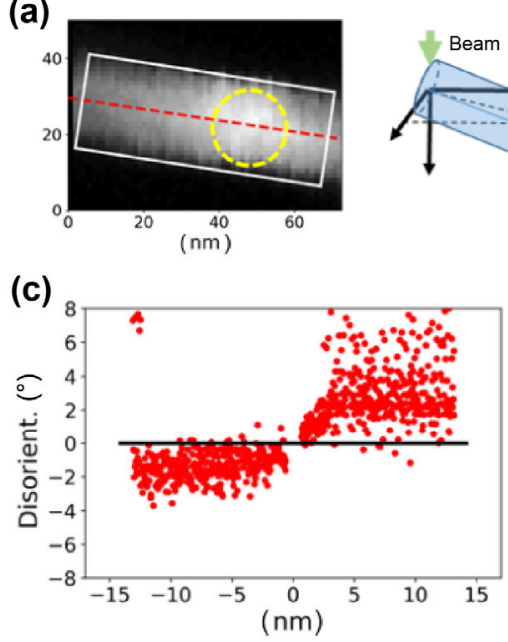

(b)

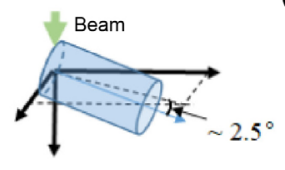

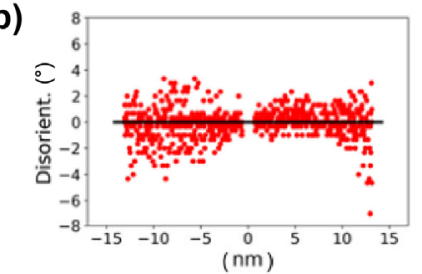

(d)

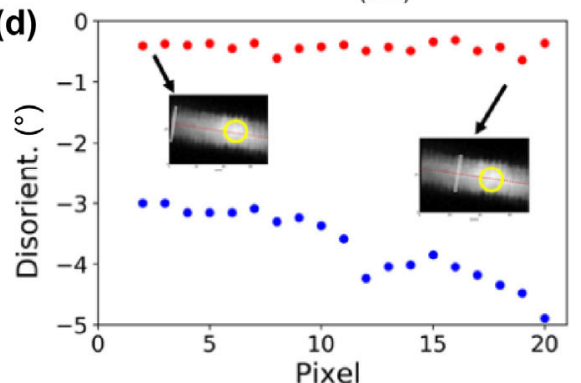

Figure 5 Analysis of the screw dislocation strain field with higher spatial resolution. (a) VADF image of the InP nanowire and schematic draw of the nanowire orientation in relation to the sample coordinates, and the round circle indicates a sample region oriented very close to [11 $\overline{2} 0]$ zone axes. (b) and (c) Misorientation of the wurtzite $c$-axis in relation to wire geometrical axis in the plane perpendicular and parallel to the electron beam respectively; we have plotted all pixels within the white rectangle in (a), except the points very close to the dislocation core. (d) Actual PED measured angle in relation to the sample plane (perpendicular to the electron beam) for each side across the dislocation line (from left to right, see insets); note that angle changes along the wire as a function of the distance to the region oriented close to a zone axis suggesting the presence of dynamical diffraction effects (see text for explanations).

(a)

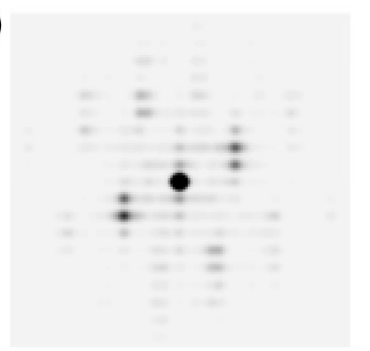

(b)

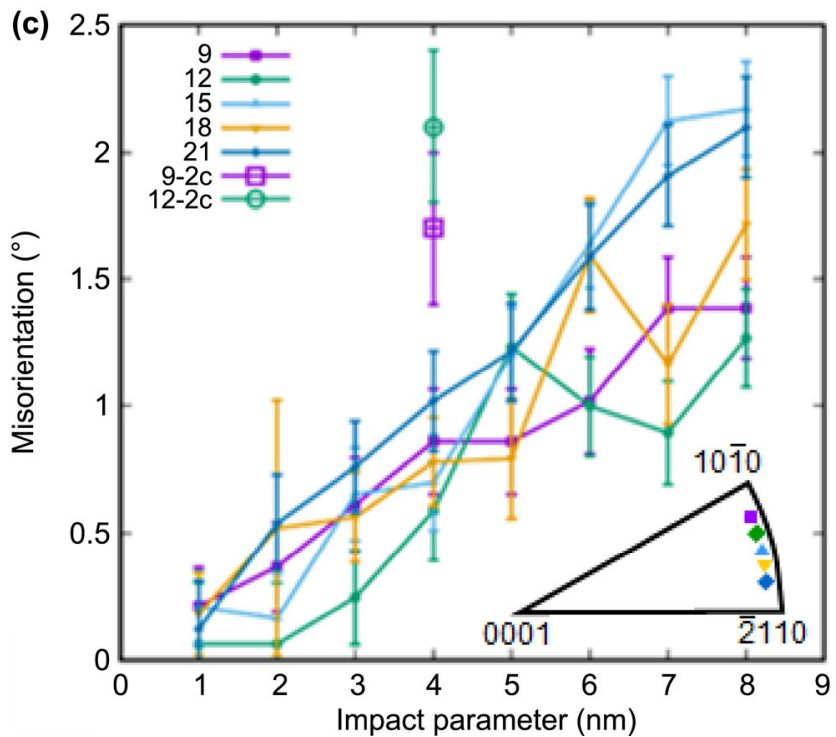

Figure 6 Dynamical diffraction simulations of PED patterns from the InP nanowire prepared for (a) a surface normal at $9^{\circ}$ from the (1010) pole at an impact parameter of $2 \mathrm{~nm}$ from the dislocation position $\left(0.5^{\circ}\right.$ precession angle) and (b) the same surface normal but at an impact parameter of $8 \mathrm{~nm}$. (c) Summary of the calculated misorientations for 8 impact parameters along 5 different surface normals (the legend numbers 9-21 indicate the angle, in degrees, between the (1010) pole and the surface normal, the inset shows the position of the selected crystal orientation in a pole figure). The open markers show the calculated misorientation for simulated patterns with a Burger's vector of $2|\boldsymbol{c}|$ at different orientations. Error bars indicate the effect of the finite spacing of the simulated patterns in the library and the resultant range of misorientations for the simulated patterns.

Measurement of the helicoidal geometry that arises from the dislocation in the wire is a more direct method to characterize the dislocation but as we have seen this is not without its difficulties. The twisted NW presents a range of different crystalline orientations along the electron beam path. The first material encountered must be at the surface of the NW, so lies at the maximum radial distance from the dislocation core. However as the electron traverses to the central plane of the NW ( $x-z$ in the schema in Fig. 1) the radial distance to the dislocation decreases. As a result, along its trajectory the electron encounters material with continually varying $c$-axis normal direction $(N)$ and this is further complicated by the continuous change in the azimuthal position of the material being traversed. Given the nature of electron scattering it is perhaps predictable that the diffracted beams from each portion of the crystal should interact dynamically. What is interesting is that the results in this study indicate that for arbitrary positions on the wire the material first encountered by the electron beam (with the smallest vertical component of misorientation) seems to contribute the greatest amount to the measured diffraction pattern. This explains the tendency towards zero vertical misorientation being measured at an impact parameter of zero in both the simulated and experimental misorientation results.

The exception to this would seem to be when material close to a major zone-axis orientation is encountered by the electron beam. In these situations, it would seem that the strong Bragg conditions found in these orientations tend to provide the major contribution to the measured diffraction pattern. This would explain the inability to determine a smooth orientation change close to the zone axes (as seen in Fig. S5 in the ESM).

By simulating the diffraction from the twisted NW, rather than rely on direct measurements of orientation from the diffraction pattern it is however possible to verify the Burger's vector magnitude in the NW independently of the Eshelby equation for twist rate.

\section{Conclusions}

This manuscript presents a detailed analysis of structural distortions present in wurtzite InP NWs (30-40 nm in diameter) containing a screw dislocation and a wire lattice torsion (Eshelby twist). As strain can strongly modify electronic properties of InP NWs [30,31], a 
precise characterization of the crystalline structure is necessary. Our results suggest that while the measured Eshelby twist rate is slightly higher (ca. 1.2 $\times$ ) than expected from classical elastic theory and classical energetic considerations, our inability to measure precisely the diameter (and area) of material supporting the strain field means that we estimate this discrepancy to be within the experimental error. However, the dislocation was independently characterized using the radial strain field to confirm the Burger's vector, while simultaneously gathering parameters such as direction, sense and handedness of the screw dislocation with high confidence. The modulus of the Burgers vector was found to be compatible with $|\boldsymbol{c}|$, and higher values such as $|\boldsymbol{B}|=2|\boldsymbol{c}|$ could be excluded.

These experiments illustrate the importance of spatially resolved PED data to characterize subtle structural deformations in individual NWs. The effect of using disoriented wire regions to analyze the fine details of the strain field has been shown. The observed crystal attributes was measured with high precision combining real and reciprocal space information. It is important to emphasize that this study involved a rather beam sensitive material (InP) and electron irradiation has been minimized by the fact the PED analysis does not require a carefully oriented sample.

We have applied precession diffraction very close to its sensitivity and precision limits and, experimental results were compared with full dynamical diffraction simulations to understand the observed crystal misorientations. There is the possibility of improving the precision of the template matching algorithm by selecting the regions to specifically avoid zone axis conditions. Further improvements in precision could be achieved by using an axial camera to reduce PED pattern distortions and using more advanced dynamical diffraction calculations to prepare the libraries of patterns used for orientation matching $[32,33]$, which would accurately utilize the intensities of diffraction spots in addition to their position.

\section{Methods}

\subsection{Nanowire growth}

The In(Ga)P NWs were grown using the vapor-liquid-solid (VLS) mechanism $[26,34]$ in a chemical beam epitaxy (CBE) reactor. Thermally decomposed phosphine (PH3), trimethyl Indium (TMI) and triethyl Gallium (TEGa) were used as precursors, with $\mathrm{H}_{2}$ as carrier gas [25]. Physically deposited Au nanoparticles have been used as catalysts on nominal [001] GaAs substrates. The particles have been synthesized by thermal evaporating $\mathrm{Au}$ in a low pressure (tens of mbar) Ar atmosphere [35]; the substrate was kept at 300$350{ }^{\circ} \mathrm{C}$ during deposition. The sample displaying a significant amount of wires containing screw dislocations was produced during growth experiments attempting to synthesize InGaP NWs. Although TEGa was introduced in the growth chamber, routine X-ray TEM analysis has not detected any Ga presence in thin NW regions, although Ga could be identified in the laterally grown shell in thicker NWs. The wires were transferred by gently scrapping a lacey carbon grid on the substrate; this procedure breaks the NWs from the substrate and they become attached to the carbon film. The wires containing the screw dislocation could be identified because of their remarkable length $[25,36]$ up to $10 \mu \mathrm{m}$ and where the wires axis follows a [0001] growth direction.

\subsection{Electron microscopy measurements}

Electron diffraction experiments were realized in Philips CM300 field emission gun (FEG) TEM/STEM microscope operated at $200 \mathrm{kV}$, a convergent beam geometry was used with a convergence semi-angle of $1.4 \mathrm{mrad}$, and TEM optics was adjusted to achieve probes sizes as a function of experiment aim. InP is quite sensitive to electron irradiation in the TEM, so we used a rather wide probe (diameter $>10 \mathrm{~nm}$, scan pixel size $4 \mathrm{~nm}$ ) to measure the crystal rotation where a highest spatial resolution was not required. In contrast, the structural deformation associated with the dislocation was studied using a smaller probe $(\sim 1 \mathrm{~nm}$, scan pixel size $1.25 \mathrm{~nm})$. The measurements were run using a Nanomegas DIGISTAR system to drive the pre- and post-sample deflector coils allowing the precession rocking and de-rocking as well as the raster scan over the sample. Diffraction patterns were recorded by recording the microscope phosphor viewing screen using an external high speed CCD camera binned to $144 \times 144$ pixels for twist assessment. Precession diffractions patterns were acquired at $0.5^{\circ}$ precession angle and $50 \mathrm{~ms}$ exposure per image with a precession frequency of $100 \mathrm{~Hz}$. The HSR-PED data sets were processed to obtain the orientation mapping by means of the ASTAR pattern matching software and libraries describing at least 150 orientations along each edge of the stereographic triangle were generated from the InP wurtzite crystal structure to achieve the needed angular resolution for crystal rotation measurements.

HSR-PED experiments (shown in Fig. 5) were preformed to optimize the spatial and angular resolution to determine $c$-axis misorientation. We scanned smaller areas $(60 \times 60$, pixel $1.25 \mathrm{~nm})$ with a nm wide probe, and slightly tilted the wire a few degrees off $\left(\sim 2.5^{\circ}\right)$ in order to avoid strong dynamical conditions and, defocusing effects associated to sample height changes. The full camera capacity $(512 \times 512$ pixels patterns $)$ was used and Poisson noise reduced using recent methods based on those proposed by Azzari et al. [37-40] which includes both the Anscombe transformation and collaborative denoising based on the BM3D approach. Figure S7 in the ESM shows the effect of the denoising of a diffraction pattern, the noise appears significantly reduced and at the same time the peak intensity seems to be conserved. This approach has recently been applied to denoise atomic resolution images used in the tomographic reconstruction of FePt nanoparticles [41].

Finally, crystal orientations were derived by the conventional PED matching procedure using larger libraries of simulated diffraction patterns (data bank including 300-350 orientation steps along reduced zone edges).

\subsection{Mapping screw dislocation field and Eshelby twist by HSR-PED}

The orientation maps were derived using the ASTAR software (template matching) in order to yield the three Euler angles $\left(\varphi_{2}, \Phi\right.$, $\left.\varphi_{1}\right)$ for each pixel. Subsequently, a rotation matrix was built using the angles that are provided in Bunge notation [42]; this step and all further processing discussed below were performed using a homemade software in Python. This was used for two analyses: (a) the third matrix column represents the crystal reciprocal vector $(h k l)$ along the sample normal (ND) (in TEM experiment, it is considered as the electron beam direction) to study the crystal torsion/twist; (b) in analogy, the third matrix row represent the cosine vectors $[\boldsymbol{x}, \boldsymbol{y}, \boldsymbol{z}]$ indicating the reciprocal vector ( $c$-axis) orientation in sample coordinates to analyze the screw dislocation strain field.

In contrast to polycrystalline samples, where Euler angles may present discrete jumps at grain boundaries, the twisted NWs must show a smooth axial evolution of the crystal rotation as a function of the axial position (the Eshelby twist). This smoothness has been used to correct eventual occurrence of the " $180^{\circ}$ ambiguity" that can appear in the pattern recognition methodology [12]. Due to the high symmetry of the InP wurzite structure ( $P 6_{3} m c$ space group [20]), we were forced to correct other possible ambiguities associated with the equivalent positions in the basal plane in order to get consistent crystal rotation evolution along the wire from the ASTAR matching template procedure.

Since each pixel in the NW PED map yields a different crystal orientation. The orientation exactly at the wire axis (on the dislocation core) was derived by averaging the central pixel and the two 
neighboring ones in opposite transversal radial direction. Then the measurement will yield the wire axis direction (i.e. actually the wire geometrical axis) that we will adopt as an "internal" reference

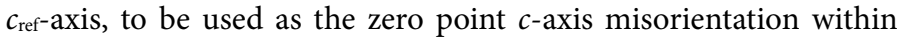
the wire.

It is important to emphasize that performing HSR-PED experiment in nanostructures whose crystallographic orientation is close to a zone axis should be avoided. The uncertainty on the determination of orientation increases in these cases $[12,43]$; for example the NW in Fig. 4(a) has been selected because its axis is not perpendicular to the beam and about $5^{\circ}-15^{\circ}$ out of the sample plane, see pole figure in Fig. 4(b). If similar data is taken with the wire axis (wurtzite $c$-axis) perpendicular to the electron beam, the increase in misorientation uncertainties in regions close to zone axis become significant (see Fig. S5 in the ESM). The origin of this effect may be associated both to strong dynamical diffraction effects and also to the PED template matching procedure which is not appropriate to analyze diffraction pattern where many diffractions spots may show saturated intensity.

\subsection{Determination of the $3 \mathrm{D}$ wire shape from PED data}

In order to get the location of the wire axis (or dislocation core) in the PED map, virtual bright field (VBF) or virtual dark field (VDF) images were generated from the individual diffraction patterns [44] Such images provide a real space map of the sample. The image was fitted with a polynomial function, yielding the position of the wire axis in our orientation map (Fig. 4(a)); also, its derivative provides the in-plane (perpendicular to the electron beam) direction of the crystal $c$-axis in the image pixel. As expected this in-plane direction reproduces very well the wire axis direction derived from PED data, except for pixels with high noise/systematic errors (ex. close to zone axis [44]) or displaying the $180^{\circ}$ ambiguity [12] (which can then be corrected).

Additionally, these wires are rather thin and they tend to be curved on the TEM grids (Fig. 2(a)). Once the in-plane wire shape is established from the image, the elevation angle of the $c$-vector for each pixel can be obtained from the PED derived Euler angles and rotation matrix. This information can be used to infer the $3 \mathrm{D}$ curvature of the wire by adding the vector representing the $c$-axis direction pixel by pixel along the wire axis (see schematic draw in Fig. S3 in the ESM). This procedure allows us to determine experimentally the actual $3 \mathrm{D}$ curve followed by the NW axis. This is very important because although we obtain bi-dimensional map/images, we are able to calculate with much better precision the actual NW axial distance changes between pixels. This improves the measurement of rotation rate associated with the Eshelby twist.

The access to the 3D shape of the wire, provides a series of very useful general information, as for example the sample height. In HRS-PED, the alignment procedure aims to join three points: the precession pivot points, the descan pivot point and the sample plane $[45,46]$ to obtain a high spatial resolution. When performing scans over areas of several square microns, the sample height may change, which may induce significant beam broadening due to defocus of the precessing electron probe. Figure S4 in the ESM, shows measurement for two different NW regions where the measured sample height changes are larger (in the $200-400 \mathrm{~nm}$ range). Considering that PED precession angle in $0.5^{\circ}$ these defocus values may induce the increase of the electron probe size by several Angströms or even nanometers. We have obtained the wire diameter by fitting a Gaussian curve to intensity profiles along the wire axis (see bottom sector of Figs. S4(a) and S4(b) in the ESM). In fact, the VADF images show that the wire width (represented by the Gaussian full width at half maximum, FWHM) increases in agreement with the deduced broadening for wire displayed in Fig. S4(a) in the ESM. In contrast,
Fig. S4(b) in the ESM shows that the wire diameter increase can be as large as $20 \mathrm{~nm}$ for a height change of approximatively $200 \mathrm{~nm}$; this width increase cannot be accounted for probe defocusing only. A careful look at the NW 3D shape indicates the formation of an elbow above the sample plane. This strongly bent region is probably detached from the sample grid and may show significant vibration amplitude, which would induce the observed image broadening.

\subsection{Multislice simulation of HSR PED patterns}

A model of the InP NW was prepared comprising $~ 13,500$ atoms arranged initially as a perfect hexagonal prism of InP, $22 \mathrm{~nm}$ in diameter and 2 unit cells long with the same axis system as Fig. 1. The dislocation helicoid was then introduced altering the $z$-positions of the atoms depending on their $x-y$ coordinate. The model could be rotated about the $z$-axis to present the atomic arrangement at any orientation (equivalent to addressing a given position along the Eshelby twisted NW). To avoid a situation where a strong systematic row of reflections was in all patterns, and to best match the experimental data, the $z$-axis was also tilted $2.5^{\circ}$ downward in $y$ (as in Fig. 5). Finally square sections of approximately $1 \mathrm{~nm}$ by $1 \mathrm{~nm}$ were extracted from the model and used as the input for multislice simulations [47] after separating into 20 slices along the section thickness. While perfect periodic boundary conditions cannot be achieved for such an approach the best possible periodicity was chosen and the relatively large cell meant that artefacts were minimized. The PED simulations were produced by summing 200 individual diffraction patterns for evenly spaced azimuthal trajectories with a beam tilt $0.5^{\circ}$ away from the sample surface normal.

Patterns were simulated for 7 different surface normals between $(10 \overline{10})$ and $(11 \overline{2} 0)$ poles and for impact parameters starting from $0 \mathrm{~nm}$ (at the dislocation core) and then out in $1 \mathrm{~nm}$ steps to $8 \mathrm{~nm}$. Examples for the surface normal $9^{\circ}$ from the (1010) pole at an impact parameter of 1 and $8 \mathrm{~nm}$ are shown in Figs. 6(a) and 6(b) respectively. The imperfect boundary conditions in the simulation manifest as a slight horizontal streaking in the patterns but the location of the Bragg peaks is not affected by this effect. These patterns were then assigned an orientation using the same pattern matching procedure as the experimental data and misorientations were calculated against the orientation of the simulated pattern at an impact parameter of $0 \mathrm{~nm}$.

\section{Acknowledgements}

We thank Dr Z. Saghi for taking the ADF-STEM images of the twisted wires. D. U. acknowledges financial support from the Brazilian Agencies FAPESP (No. 2014/01045-0) and CNPq (No. 302767/2012-6). A. E. acknowledges funding from the Royal Society. P. A. M. acknowledges financial support from European Research Council through grant 291522-3DIMAGE and the EPSRC grant number EP/R025517/1. M. A. C. acknowledges financial support from FAPESP (Nos. 2013/02300-1 and 2013/10957-0) and CNPq (No. 479486/ 2012-3). L. H. G. T. and P. A. M. acknowledge funding from the European Union Seventh Framework Programme (FP7/2007-2013) under Grant Agreement No. 312483 (ESTEEM2).

Electronic Supplementary Material: Supplementary material (further details of the crystal structure of the wurtzite InP nanowires, determination of nanowire width, determination of the 3D shape of the NW, the effect of the 3D shape of the wire on the image focusing, HSR-PED experiment for well oriented crystals, linear averaging of the misorientation along electron trajectory, Poisson denoising of PED patterns by the Anscombe transformation and videos of the experimental data) is available in the online version of this article at https://doi.org/10.1007/s12274-019-2328-5. 
Open Access This article is licensed under a Creative Commons Attribution 4.0 International License, which permits use, sharing, adaptation, distribution and reproduction in any medium or format, as long as you give appropriate credit to the original author(s) and the source, provide a link to the Creative Commons licence, and indicate if changes were made.

The images or other third party material in this article are included in the article's Creative Commons licence, unless indicated otherwise in a credit line to the material. If material is not included in the article's Creative Commons licence and your intended use is not permitted by statutory regulation or exceeds the permitted use, you will need to obtain permission directly from the copyright holder.

To view a copy of this licence, visit http://creativecommons.org/ licenses/by/4.0/.

\section{References}

[1] Lieber, C. M. Nanoscale science and technology: Building a big future from small things. MRS Bull. 2003, 28, 486-491.

[2] Yang, P. D. The chemistry and physics of semiconductor nanowires. MRS Bull. 2005, 30, 85-91.

[3] Agarwal, R. Heterointerfaces in semiconductor nanowires. Small 2008, 4, 1872-1893.

[4] Erni, R. Aberration-Corrected Imaging in Transmission Electron Microscopy: An Introduction; Imperial College Press: London, UK, 2015.

[5] Williams, D. B.; Carter, C. B. Transmission Electron Microscopy-A Textbook for Materials Science; Springer: Boston, MA, 2009.

[6] Pennycook, S. J.; Nellist, P. D. Scanning Transmission Electron Microscopy; Springer: New York, 2011.

[7] Carter, B.; Williams, D. B. Transmission Electron Microscopy: Diffraction, Imaging, and Spectrometry; Springer: Switzerland, 2016.

[8] Hammond, C. The Basics of Crystallography and Diffraction, 3rd ed.; Oxford University Press: Oxford, 2009.

[9] Vincent, R.; Midgley, P. A. Double conical beam-rocking system for measurement of integrated electron diffraction intensities. Ultramicroscopy 1994, 53, 271-282.

[10] Eggeman, A. S.; Midgley, P. A. Precession electron diffraction. In Advanced in Imaging and Electron Physics; Hawkes, P. W., Eds.; Elsevier/Academic: Amsterdam, 2012; pp 1-63.

[11] Midgley, P. A.; Eggeman, A. S. Precession electron diffraction-A topical review. IUCrJ 2015, 2, 126-136.

[12] Rauch, E. F.; Véron, M.; Portillo, J.; Bultreys, D.; Maniette, Y.; Nicolopoulos $\mathrm{S}$. Automatic crystal orientation and phase mapping in TEM by precession diffraction. Microsc. Anal. 2008, 22, S5-S8.

[13] Béché, A.; Rouvière, J. L.; Barnes, J. P.; Cooper, D. Strain measurement at the nanoscale: Comparison between convergent beam electron diffraction, nano-beam electron diffraction, high resolution imaging and dark field electron holography. Ultramicroscopy 2013, 13, 10-23.

[14] Cooper, D.; Bernier, N.; Rouvière, J. L. Combining 2 nm spatial resolution and $0.02 \%$ precision for deformation mapping of semiconductor specimens in a transmission electron microscope by precession electron diffraction. Nano Lett. 2015, 15, 5289-5294.

[15] Cooper, D.; Denneulin, T.; Bernier, N.; Béché, A.; Rouvière, J. L. Strain mapping of semiconductor specimens with $\mathrm{nm}$-scale resolution in a transmission electron microscope. Micron 2016, 80, 145-165.

[16] Hÿtch, M. J.; Snoeck, E.; Kilaas, R. Quantitative measurement of displacement and strain fields from HREM micrographs. Ultramicroscopy 1998, 74, 131-146.

[17] Hÿtch, M. J.; Houdellier, F. Mapping stress and strain in nanostructures by high-resolution transmission electron microscopy. Microelectron. Eng. 2007, 84, 460-463.

[18] De Graef, M. Introduction to Conventional Transmission Electron Microscopy; Cambridge University Press: Cambridge, 2003.

[19] Zuo, J. M.; Spence, J. C. H. Advanced Transmission Electron Microscopy: Imaging and Diffraction in Nanoscience; Springer: New York, 2017.

[20] Dacal, L. C. O.; Cantarero, A. Ab initio electronic band structure calculation of InP in the wurtzite phase. Solid State Commun. 2011, 151, 781-784.

[21] Eshelby, J. D. Screw dislocations in thin rods. J. Appl. Phys. 1953, 24, $176-179$.
[22] Eshelby, J. D. The twist in a crystal whisker containing a dislocation. Philos. Mag. 1958, 3, 440-447.

[23] Zhu, J.; Peng, H. L.; Marshall, A. F.; Barnett, D. M.; Nix W. D.; Cui, Y. Formation of chiral branched nanowires by the Eshelby Twist. Nat. Nanotechnol. 2008, 3, 477-481.

[24] Bierman, M. J.; Lau, Y. K. A.; Kvit, A. V.; Schmitt, A. L.; Jin, S. Dislocation-driven nanowire growth and Eshelby Twist. Science 2008, 320, 1060-1063.

[25] Tizei, L. H. G.; Craven, A. J.; Zagonel, L. F.; Tencé, M.; Stéphan, O.; Chiaramonte, T.; Cotta, M. A.; Ugarte D. Enhanced Eshelby twist on thin wurtzite InP nanowires and measurement of local crystal rotation. Phys. Rev. Lett. 2011, 107, 195503.

[26] Hiruma, K.; Yazawa, M.; Katsuyama, T.; Ogawa, K.; Haraguchi, K.; Koguchi, M.; Kakibayashi H. Growth and optical properties of nanometer-scale GaAs and InAs whiskers. J. Appl. Phys. 1995, 77, 447-462.

[27] Morin, S. A.; Bierman, M. J.; Tong, J.; Jin, S. Mechanism and kinetics of spontaneous nanotube growth driven by screw dislocations. Science 2010, $328,476-480$.

[28] Wu, H. Y.; Meng, F.; Li, L. S.; Jin, S.; Zheng, G. F. Dislocation-driven CdS and CdSe nanowire growth. ACS Nano 2012, 6, 4461-4468.

[29] Meng, F.; Morin, S. A.; Forticaux, A.; Jin, S. Screw dislocation driven growth of nanomaterials. Acc. Chem. Res. 2013, 46, 1616-1626.

[30] Chauvin, N.; Mavel, A.; Patriarche, G.; Masenelli, B.; Gendry, M.; Machon, D. Pressure-dependent photoluminescence study of wurtzite InP nanowires. Nano Lett. 2016, 16, 2926-2930.

[31] De Luca, M.; Polimeni, A. Electronic properties of wurtzite-phase InP nanowires determined by optical and magneto-optical spectroscopy. Appl. Phys. Rev. 2017, 4, 041102.

[32] Palatinus, L.; Corrêa, C. A.; Steciuk, G.; Jacob, D.; Roussel, P.; Boullay, P.; Klementová, M.; Gemmi, M.; Kopeček, J.; Domeneghetti, M. C. et al. Structure refinement using precession electron diffraction tomography and dynamical diffraction: Tests on experimental data. Acta Cryst. B 2015, 71, 740-751.

[33] Palatinus, L.; Petříček, V.; Corrêa, C. A. Structure refinement using precession electron diffraction tomography and dynamical diffraction: Theory and implementation. Acta Cryst. A 2015, 71, 235-244.

[34] Wagner, R. S.; Ellis, W. C. Vapor-liquid-solid mechanism of single crystal growth. Appl. Phys. Lett. 1964, 4, 89-90.

[35] Hayashi, T.; Ohno, T.; Yatsuya, S.; Uyeda, R. Formation of ultrafine metal particles by gas-evaporation technique. IV. Crystal habits of iron and FCC metals, Al, Co, Ni, Cu, Pd, Ag, In, Au and Pb. Jpn. J. Appl. Phys. 1977, 16, 705-717.

[36] Frank, F. C. Capillary equilibria of dislocated crystals. Acta Cryst. 1951, 4, 497-501.

[37] Anscombe, F. J. The transformation of Poisson, binomial and negativebinomial data. Biometrika 1948, 35, 246-254.

[38] Mäkitalo, M.; Foi, A. A closed-form approximation of the exact unbiased inverse of the anscombe variance-stabilizing transformation. IEEE Trans. Image Process. 2011, 20, 2697-2698.

[39] Mäkitalo, M.; Foi, A. Optimal inversion of the anscombe transformation in low-count Poisson image denoising. IEEE Trans. Image Process. 2011, 20, 99-109.

[40] Azzari, L.; Foi, A. Variance stabilization for noisy+estimate combination in iterative Poisson denoising. IEEE Signal Process. Lett. 2016, 23, 1086-1090.

[41] Yang, Y.; Chen, C. C.; Scott, M. C.; Ophus, C.; Xu, R.; Pryor, A.; Wu, L.; Sun, F.; Theis, W.; Zhou, J. H. et al. Deciphering chemical order/disorder and material properties at the single-atom level. Nature 2017, 542, 75-79.

[42] Kocks, U. F.; Tomé, C. N.; Wenk, H. R. Texture and Anisotropy; Cambridge University Press: Cambridge, 1998.

[43] Morawiec, A.; Bouzy, E.; Paul, H.; Fundenberger, J. J. Orientation precision of TEM-based orientation mapping techniques. Ultramicroscopy 2014, 136, 107-118.

[44] Rauch, E. F.; Véron, M. Virtual dark-field images reconstructed from electron diffraction patterns. Eur. Phys. J. Appl. Phys. 2014, 66, 10701.

[45] Liao, Y. F.; Marks, L. D. On the alignment for precession electron diffraction. Ultramicroscopy 2012, 117, 1-6.

[46] Barnard, J. S.; Johnstone, D. N.; Midgley, P. A. High-resolution scanning precession electron diffraction: Alignment and spatial resolution. Ultramicroscopy 2017, 174, 79-88.

[47] Eggeman, A. S.; London, A.; Midgley, P. A. Ultrafast electron diffraction pattern simulations using GPU technology. Applications to lattice vibrations. Ultramicroscopy 2013, 134, 44-47. 